\title{
Layered Animation of Captured Data
}

\author{
Wei Sun, Adrian Hilton, Raymond Smith and John Illingworth \\ Centre for Vision, Speech and Signal Processing \\ University of Surrey, Guildford GU2 5XH, UK
}

a.hilton@surrey.ac.uk http://www.ee.surrey.ac.uk/Research/VSSP/3Dvision

\begin{abstract}
This paper proposes a novel technique for building layer animation models of real articulated objects from 3D surface measurement data. Objects are scanned using a hand-held 3D sensor to acquire 3D surface measurements. A novel geometric fusion algorithm is presented which enables reconstruction of a single surface model from the captured data. This algorithm overcomes the limitations of previous approaches which cannot be used for hand-held sensor data as they assume that measurements are on a structured planar grid. The geometric fusion introduces the normal-volume representation of a triangle to convert individual triangles to a volumetric implicit surface.

A layered model is constructed to animate the reconstructed high-resolution surface. The model consists of 3 layers: skeleton for animation from key-frame or motion capture; low-resolution-controlmodel for real-time mesh deformation; and high-resolution model to represent the captured surface detail. Initially the skeleton model is manually placed inside the low-resolution control model and high-resolution scanned data. Automatic techniques are introduced to map both the control model and captured data into a single layered model. The high-resolution captured data is mapped onto the lowresolution control model using the normal-volume representation. The resulting model enables efficient, seamless animation by manipulation of the skeleton whilst maintaining the captured high-resolution surface detail. The animation of high-resolution captured data based on a low-resolution generic model of the object opens up the possibility of rapid capture and animation of new objects based on libraries of generic models.
\end{abstract}

Supported by EPSRC Advanced Fellowship AF/97/2531 and EPSRC Grant GR/89518 'Building Realistic Models for Virtual Reality and Animation' 


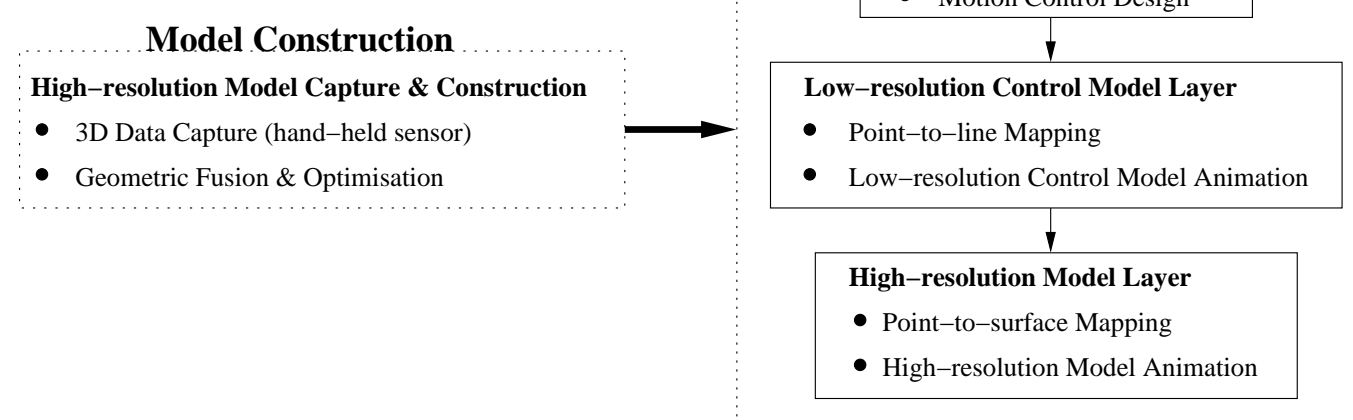

Figure 1: System overview

\section{Introduction}

One fundamental difficulty in 3D-computer animation is building 3D models that are frequently required to have not only high-detail and realistic surfaces but also believable deformations. Advances in 3D sensor technology have supplied an efficient way to capture photo-realistic 3D models of real objects. However, how to animate such kind of captured models is rarely studied. Reconstructed surface models are usually composed of a dense unstructured polygonal mesh representing the surface detail of the object at the resolution and accuracy of the sensor. "Such meshes are notoriously expensive to store, transmit, render, and are awkward to animate" [Thalmann et al., 1996]. The goal of this research is to enable rapid reconstruction of models suitable for animation from captured data.

Figure 1 shows an overview of our system. The system consists of two parts: model construction and layered animation. The function of the first part is to obtain a single triangulated 3D surface mesh with high surface detail from real objects. A hand-held sensor system is used for data acquisition from 3D models (section 3.1). The normal-volume representation concept is used for the fusion of overlapping surface measurements into a single mesh surface model (section 3.2). A high-resolution triangulated surface representation is generated using a local implicit surface based geometric fusion algorithm (section 3.3).

To animate reconstructed surface models from captured data realistically and efficiently, a layered model is built in which each layer performs different animation tasks. The layered model allows an animator to manipulate the low-resolution model in real-time and render the full surface detail off line to achieve full realism. This paper is an extension of previously reported work in progress [Sun et al., 1999] to present in detail the complete chain from captured data to an animated model. This framework addresses limitations 
of our previous work in building and realistically animating the layered model.

The lowest layer here is the skeleton layer. A hierarchical skeleton structure is fitted interactively to the high and low resolution mesh models, as introduced in section 4.1. The fitted skeleton model gives the joint position information and can be animated using common animation techniques such as key-frame, inverse kinematics, physics-based models or motion capture techniques. This greatly simplifies the process of animating the captured model. The skeleton motion provides input for all other layers.

The middle layer is a low-resolution control model. Although the skeleton alone is sufficient for motion control purposes, it is often too simple to be used as a representation of an object. On the other hand the high-resolution model is prohibitively expensive to work with. Therefore it is desirable to introduce an intermediate layer. The control model is a generic representation of the object with the same basic shape and topology as the captured data but a low polygon count. It could be a box-like model as in Kinetix's Biped for 3D Studio MAX or more complicated models such as those available from Viewpoint Data Labs [Datalabs, 1996]. Such models are structured to achieve smooth and efficient animation of the object surface. Firstly, in the control model layer, a skeleton is manually located inside the model. A point-to-line mapping is then used to automatically map the low-resolution model onto the skeleton (section 4.2). In this paper we also introduce a new point-to-line mapping algorithm which enables full geometric deformation of the sphere including twist based on the skeleton motion. This overcomes limitations of our previous pointto-line mapping [Sun et al., 1999] which did not allow control model deformation based on twist motion. A seamless deformation scheme is developed to animate the control model based on the parameterisation obtained from the point-to-line mapping. For greater realism the layered model could be animated using alternative deformation scheme such as FFD or physical-based modelling as discussed in section 2.

The high-resolution captured data model is automatically mapped onto the low-resolution control model using the point-to-surface mapping (section 4.3). This mapping allows the high-resolution data to be mapped to an control model of arbitrary shape and topology. Normally the control model is structured to achieve smooth and efficient animation of the object surface. The high-resolution model can then be animated efficiently based on the control model animation using a continuous deformation scheme (section 5.2). The result is that the high-resolution captured model is introduced at the rendering stage to achieve both realistic modelling and animation of the captured object.

This approach provides a novel technique for building animated models from captured surface measurements. The process is considerably more efficient and less time consuming than current manual schemes used by animation companies. An important contribution of this research is to enable captured data to be 
mapped onto a pre-defined generic object models which have been optimised for realistic and efficient animation. The resulting layered model gives an efficient realistic animation of the fine surface detail while providing a low-resolution control structure for real-time animation control and visualisation.

\section{Background}

There has been extensive commercial interest in developing techniques for measurement of surface shape in many fields for 3D model generation. Commercial 3D sensors have become widely available in recent years for capturing surface measurement data of objects, including objects as complex as the human body. This section reviews previous work on model building from captured data and techniques for model animation.

\subsection{Model Building}

Manual techniques have been used extensively to capture object shape using a touch probe sensor. This approach to digitising 3D shape has been used to generate model libraries [Datalabs, 1996]. There has also been extensive interest in using active optical 3D surface measurement technologies which project a structured light onto the object surface and use triangulation measure the distance. Such systems enable high-accuracy non-contact digitisation of the object surface.

The majority of sensors capture 3D measurements with respect to a 2D planar or cylindrical grid. This approach requires multiple scans to be taken in order to digitise the entire object surface. Research has addressed the problem of reconstructing a single surface model from the captured 3D surface measurements [Curless and Levoy, 1996, Hilton et al., 1996, Hoppe et al., 1992, Rutishauser et al., 1994, Turk and Levoy, 1994, Soucy and Laurendeau, 1995]. Recently reconstruction techniques based on fusion of overlapping data using a volumetric implicit surface representation have enabled rapid and reliable reconstruction of highresolution models of complex objects with an arbitrary topology and geometry [Curless and Levoy, 1996, Hilton et al., 1998]. The general problem of surface reconstruction of unknown objects from unstructured 3D point remains an open-problem [Bittar et al., 1995, Edelsbrunner and Mucke, 1994, Hoppe et al., 1992, Mencl, 1995, Mencl and Muller, 1998].

We use a hand-held 3D sensor which does not constrain the 3D measurements to lie on a 2D planar or cylindrical grid. This sensor has the advantage that it can be freely moved around the object to position the sensor for capture of complex geometric features such as a characters mouth. As the resulting 3D surface measurement data is not structured on a regular grid previous surface reconstruction algorithms cannot be 
applied. Fusion of measurements from a hand-held sensor presents an additional challenge that the sensor accuracy is currently an order of magnitude lower than conventional range image sensors. This is due to the requirement for a low cost six degree-of-freedom device to measure the spatial position of the sensor.

In this paper we introduce an extension to previous implicit surface based reconstruction approaches [Hilton et al., 1996, Hilton et al., 1998] which enables fusion of hand-held sensor data. This is the first geometric fusion algorithm capable of integrating surface measurements from a hand-held range sensor. This approach can be used to integrate measurements from either a conventional 2.5D range image or hand-held 3D sensor. The advantage of this approach is that it overcomes the assumption of previous volumetric integration algorithms [Curless and Levoy, 1996] that surface measurements are structured on a regular 2D planar grid with a single viewpoint. Overlapping measurements are integrated using operations in $3 \mathrm{D}$ space only. This enables integration of complex geometry and topology which was not possible with previous techniques for conventional range image sensors which are based on a 2D projection of the data [Rutishauser et al., 1994, Soucy and Laurendeau, 1995, Turk and Levoy, 1994].

\subsection{Mesh Optimisation and Surface Fitting}

Surface reconstruction results in an unstructured polygonal mesh representation of the surface. This is a highly inefficient representation as fine detail is modelled at the same resolution as smooth surface regions. A number of mesh optimisation algorithms have been proposed to post-process the redundant meshes obtained from surface reconstruction [Schroeder et al., 1992, Hoppe, 1996, Kalvin and Taylor, 1996, Soucy and Laurendea The objective of these algorithms is to automatically reduce the number of polygons while maintaining an accurate and/or realistic representation. These algorithms reduce the triangle/vertex count of the triangulation and improve uniformity of triangle shape. Some optimisation algorithms [Kalvin and Taylor, 1996] enable the geometric accuracy to be maintained within a tolerance of the original mesh. Recent mesh optimisation algorithms [Hoppe, 1996] have achieved continuous level-of-detail representation of objects which preserve both the geometry of the high-resolution mesh and its overall appearance based on surface colour, surface topology, discontinuities, normals and texture.

Although polygonal meshes provide a sufficient representation for realistic modelling and animation high-order surface representations are widely used. High-order surfaces provide a more efficient representation of smooth surface regions. Polygonal meshes or displacement maps may also be required to model the fine-surface detail [Krishnamurthy and Levoy, 1996]. Automatic reconstruction of B-spline and sub-division surface representations from reconstructed polygonal surface models has been investigated 
[Eck et al., 1995, Eck and Hoppe, 1996, Hoppe et al., 1994]. However, the resulting models from either automatic mesh-optimisation or high-order surface fitting are not directly suitable for animation. The resulting mesh vertices are not positioned to coincide with the object articulation.

As the capture process is performed on a static object in a single pose there is no captured information on the underlying articulation structure. Therefore a manual process is required to position a subset of the mesh vertices to coincide with the required articulation structure. In this paper this is achieved by manually registering a low-resolution control model containing the articulation structure with the high-resolution captured model. Automatic registration of all points on the high-resolution model with the low-resolution model is then used to enable a seamless deformable animation of the captured model. Perhaps the most closely related work to our approach is [Krishnamurthy and Levoy, 1996] where the boundaries of a lowresolution B-spline patch model are manually identified on the high-resolution captured model. Automatic mapping is then performed to obtain a B-spline representation of smooth surface regions and displacement maps to represent fine detail. This generates a model suitable for efficient animation although no animation results were presented. The principal advantage of our approach is the construction of a low-resolution control model for real-time animation and the automatic mapping and animation of the high-resolution captured surface detail.

\subsection{Model Animation}

Realistic animation of 3D articulated characters is a very challenging task involving two main aspects: realistic motion and deformation of the body. Because of the difficulties involved in each aspect, these two problems are usually investigated separately. Models used in the majority of the motion control research are skeleton based. A variety of approaches have been proposed for deformable animation of articulated characters, such as metaballs [Shen and Thalmann, 1995], free-form deformations (FFD) [Chadwick and Parent, 1989], DFFD [Mocozzet and Mangnenat Thalmann, 1997], implicit surfaces [Bloomenthal and Wyvill, 1990], hierarchical B-splines [Forsey, 1991] and physics-based deformable models [Turner and Gobbetti, 1998]. All of those techniques are aimed at creating and animating synthetic computer generated characters. The method proposed in this paper enables high-resolution captured data to be mapped to an arbitrary lowresolution control model which can be animated using any of the techniques outlined above. Both realistic motion and deformation aspects of character animation are addressed in our approach. To design and control the motions of characters, a skeleton structure is fitted inside characters interactively and thereby existing motion control algorithms can be applied. 
The question of efficient and realistic deformation of a polygonal model with internal skeleton is addressed in many CG and Game packages [Lander, 1998, Maestri, 1996]. But the users are usually required to get down to the vertex level to map them to the bones manually. After mapping there may still be some stray vertices or bulging and crimping when the body segments are moved. To fix the problem the users may have to adjust the influence of the segments and reassign some errant vertices to the model.

In our method for each segment, the influence of its adjacent segments to its mapped vertices are decided by joint angles and the distance of the vertex to its adjacent joints. Once joint angles and positions of the skeleton are known, mapping and deformation are carried out automatically. Recent work [Babski and Thalmann, 1999a, Kalra and Magnenat Thalmann, 1998] demonstrated real-time seamless animation of polygonal models. In this paper we demonstrate that this type of seamless animation of the low-resolution model can be used to achieve realistic animation of high-resolution captured data.

FFD [Chadwick and Parent, 1989] is a widely used technique for mesh deformation which has been extended [Chang and Rockwood, 1994, Coquillart and Jancene, 1991, Hsu et al., 1992, Lamousin and Waggenspack, 199 MacCracken and Joy, 1996] to give improved performance. An object is embedded within a lattice of control points which defines a mapping from the lattice to the object. The object is deformed by manipulating the control lattice. To overcome the difficulty in defining arbitrarily shaped lattices and to deform the underlying geometry directly, axial [Lazarus and Coquillart, 1994] and wires [Singh and Fiume, 1998] deformation techniques have been proposed. The goal of these deformation approaches is to develop a method for sculpturing a geometric model at will. The deformation algorithm of the low-resolution control model proposed in this paper is specific to a class of deformations: how a geometric mesh model deforms realistically with its underneath rigid skeleton structure moves? This is an essential problem when animating articulated characters. The deformation of the high-resolution data model in this paper is based on the deformation of low-resolution control model, similar to that the deformation of the geometric model in FFD based on the deformation of the control lattice in FFD. The low-resolution control model can be defined as a control lattice. In practice it is often more convenient to choose an existing generic model with the same basic shape as the captured model from a 3D model library than constructing a control lattice.

Using a layered construction approach to building animated synthetic models has been addressed by many researchers[Chadwick and Parent, 1989, Kalra and Magnenat Thalmann, 1998, Thalmann et al., 1996, Turner and Gobbetti, 1998]. A layered model decomposes the complicated task of realistic animation into three independent sub-tasks: skeleton animation based on motion capture or key-frame;interactive animation visualisation and refinement using the real-time control model; and final off-line rendering of the 
full-resolution detailed surface. This allows animators to work on each subtask separately. Subtasks such as motion control and deformation of the low-resolution model can be performed using existing animation techniques.

In this work a layered model is constructed from captured data. The layered representation provides: skeleton based animation control; real-time visualisation using the control model; efficient animation of the high-resolution surface detail based on the control model; and realistic animation of the high-resolution data based on control model deformation using geometric, FFD or physics based techniques. The principal advantage of our approach is the use of a low-resolution control model for real-time animation and the automatic mapping and animation of the high-resolution captured surface detail. This framework enables rapid reconstruction of animated models from captured data.

\section{Model capture and construction}

The hand-held range sensor system used for capturing objects is briefly described in section 3.1. The normal-volume representation of a triangle is introduced in section 3.2 to transform from a surface-based mesh representation to an implicit volume representation. In section 3.3, a normal-volume based algorithm for fusion of 3D surface measurements from a hand-held sensor into a single high-resolution mesh is presented. The normal-volume representation is also used for point-to-surface mapping in section 4.3 and high-resolution model animation section 5.2.

\subsection{Model capture using a hand-held 3D sensor}

Hand-held 3D sensors for object surface measurement are a recent development. This technology consists of a laser stripe based range sensor combined with a six degree-of-freedom position sensor. It allows the user to move freely around an object to position the sensor to capture complex geometric shapes. The 3DScanners ModelMaker hand-held sensor system (www.3dscanners.com) (in Figure 2(a)) is used for data acquisition for models presented in this paper.

Surface measurements are on a series of consecutive stripes which do not form a regular grid pattern. The stripe orientation and measurement direction change as the user sweeps the sensor across the surface of the object. The action of data acquisition is similar to that of spraying the surface of the object with paint. Surface data can be rapidly acquired for the entire object surface in a few minutes using the users' knowledge of visibility to position the sensor in order to access complex regions. Alternative automatic 
A triangulated mesh $M=\{P, T\}$ is composed of a set of vertices $P=\left[\vec{v}_{0}, \ldots, \vec{v}_{i}, \ldots, \vec{v}_{N_{P}-1}\right]$ and a set of triangles $T=\left[t_{0}, \ldots, t_{j}, \ldots, t_{N_{T}-1}\right]$ where $\vec{v}_{i}=\left(x_{i}, y_{i}, z_{i}\right)$ is a point in $R^{3}$ and triangle $t_{j}=\left[\vec{v}_{r}, \vec{v}_{s}, \vec{v}_{t}\right]$ is composed of three vertices. We assume that $M$ is a simply connected manifold triangulation with no self-intersections. For each vertex, $\vec{v}_{i}$, of $M$, we define a single vertex normal vector, $\vec{n}_{i}$. Vertex normals are defined by a weighted average of the adjacent triangle normals:

$$
\vec{n}_{v_{r}}=\frac{\sum_{j \in \aleph_{r}} w_{j} \vec{n}_{t_{j}}}{\sum_{j \in \aleph_{r}} w_{j}}
$$

Where $\aleph_{r}$ is the set of indices for triangles adjacent to the $r^{t h}$ vertex. The weights $w_{j}$ are the angles of the adjacent triangles $t_{j}$ at the $r^{t h}$ vertex. Throughout this paper all triangle and vertex normals are of unit length.

\subsubsection{Surface-to-volume transformation}

We begin by defining a volumetric-envelope which encloses the region of 3-space around the mesh $M$ (Figure 3(a)) and enables transformation of the 2-manifold surface to a volumetric representation. An offset surface $M^{\prime}$ for mesh $M$ is given by displacing each mesh vertex by a distance $d_{r}$ in the vertex normal direction such that $\vec{v}_{r}^{\prime}=\vec{v}_{r}+d_{r} \vec{n}_{r}$. If we let $d_{r}$ to be a constant offset distance $d_{\text {max }}$ then the distance of all points on the offset surface $M^{\prime}$ is less than or equal to the offset distance $d_{\text {max }}$ from the original mesh $M$. The offset surface is a continuous mesh but may not be a simple manifold due to self-intersection. The offset-surface for a cross-section through a mesh is illustrated in Figure 3(b).

A volumetric-envelope around $M$ can be defined by two offset meshes $M^{+}$and $M^{-}$such that each vertex is displaced by a distance $d_{\max }$ and $-d_{\max }$ in the normal direction respectively. The space enclosed by $M^{+}$and $M^{-}$is a closed volumetric-envelope such that every point inside this region is less than $d_{\max }$ from the mesh $M$. The volumetric-envelope for a cross-section through a mesh is illustrated in Figure 3(c).

The volumetric-envelope encloses a continuous regions of space. The use of triangle vertex normals to obtain the offset-surfaces ensures that there are no gaps between adjacent triangles. For each triangle $t_{j}=\left[\vec{v}_{r}, \vec{v}_{s}, \vec{v}_{t}\right]$ in mesh $M$ the offset meshes $M^{+}$and $M^{-}$define a closed normal-volume, $V^{N}\left(t_{j}\right)$, between $t_{j}^{+}=\left[\vec{v}_{r}^{+}, \vec{v}_{s}^{+}, \vec{v}_{t}^{+}\right]$and $t_{j}^{-}=\left[\vec{v}_{r}^{-}, \vec{v}_{s}^{-}, \vec{v}_{t}^{-}\right]$, as illustrated in Figure 4(a).

Each side of the normal volume is a surface $S_{r s}$ constrained by three Vectors - the triangle edge $\vec{v}_{r s}=$ $\vec{v}_{s}-\vec{v}_{r}$ and the corresponding vertex normals $\vec{n}_{r}$ and $\vec{n}_{s}$ (normalised). If the vertex normals are equal $\vec{n}_{r}=\vec{n}_{s}$ then the surface $S_{r s}$ is a plane. Generally the normals are not equal. Therefore the surface $S_{r s}$ is 
The volumetric-envelope for mesh $M$ between offset meshes $M^{+}$and $M^{-}$is equivalent to the union of the normal volumes for all triangles $\bigcup_{j=0, N_{T}} V^{N}\left(t_{j}\right)$ where $V^{N}\left(t_{j}\right)$ is the normal volume for triangle $t_{j}$. Therefore, transforming the mesh $M$ to an approximate volumetric representation can be reduced to a incremental process of transforming each triangle $t_{j}$ into its normal-volume $V^{N}\left(t_{j}\right)$ representation.

\subsection{Geometric Fusion into a Single Model}

Surface measurement using either a conventional or hand-held range sensor produces a sets of overlapping meshes, $M_{0}, \ldots, M_{N}$, which do not exactly intersect due to measurement noise [Turk and Levoy, 1994, Soucy and Laurendeau, 1995]. Direct interpolation of the surface measurements based on Euclidean distance [Hoppe et al., 1992] fails to accurately reconstruct the local surface detail [Curless and Levoy, 1996]. Fusion of overlapping surface measurements based on the local surface topology is necessary for reliable reconstruction. Previous fusion schemes have assumed that measurements are structured on a regular grid [Curless and Levoy, 1996, Soucy and Laurendeau, 1995] as is the case for conventional range sensors. In this section we introduce a volumetric fusion algorithm based on the surface-to-volume transformation of section 3.2 which does not make this assumption. This approach enables fusion of data from a hand-held range sensor overcoming the limitations of previous volumetric [Curless and Levoy, 1996] and surfacebased [Soucy and Laurendeau, 1995] approaches.

Fusion of multiple overlapping meshes, $M_{0}, \ldots, M_{N}$, can be achieved by integrating the discrete implicit surface representations generated by the normal-volume transformation for each mesh. An arbitrary topology closed manifold surface $S$ can be represented in implicit form as an iso-surface of a spatial field function, $f(\vec{x})$, where $\vec{x}=[x, y, z]$ is any point in Euclidean space, $\Re^{3}$. Thus we can represent a surface by defining the field function $f(\vec{x})$ as the signed distance from a point, $\vec{x}$, to the nearest point on the surface giving the iso-surface $f(\vec{x})=0$ for all points on $S$ and $f(\vec{x}) \neq 0$ elsewhere. Integration of the implicit surfaces in overlapping volumetric regions results in a single implicit surface representation. This can then be polygonized to obtain a single triangulated surface model.

For each mesh $M_{i}$ we can define a closed offset envelope between $M_{i}^{+}$and $M_{i}^{-}$with offset distance $d_{\text {max }}$. Thus for each triangle $t_{j}$ we can define a normal-volume $V^{N}\left(t_{j}\right)$ and incrementally transform the mesh $M_{i}$ to a volumetric representation using the surface-to-volume transformation introduced in the previous section. In overlapping regions of surface measurements from different meshes the normal volumes

will intersect provided the maximum measurement error $e_{\max } \leq d_{\max }$. If this condition is satisfied we can combine the field functions from different meshes to obtain a single volumetric representation. 
Fusion of multiple meshes requires an 'overlap' test to determine if surface measurements from different meshes in close spatial proximity correspond to the same or different regions of the measured object surface. Definition of a robust overlap test is critical for reliable surface reconstruction as discussed in previous work [Hilton et al., 1996, Hilton et al., 1998]. As in previous work geometric constraints are used to estimate if overlapping measurements correspond to the same surface region based on:

1. Spatial proximity: distance between overlapping meshes is less than the maximum distance $d_{\max }$.

2. Surface orientation: overlapping meshes have surface normals with the same orientation $n_{r} . n_{t}>0$.

3. Measurement uncertainty: likelihood of measurement overlap based on estimates of measurement uncertainty.

Spatial proximity provides a coarse test of measurement overlap which has been used in previous work on structured range images with a single viewpoint [Curless and Levoy, 1996]. However, this test is unreliable for sharp edges and for surfaces in close proximity. Surface orientation enables reliable reconstruction of crease edges and thin surface sections for continuous implicit surface representation [Hilton et al., 1996]. The third constraint depends on our ability to reliably estimate measurement uncertainty. For conventional range images this can be estimated from the relative orientation of the surface normal and sensor viewpoint [Turk and Levoy, 1994, Soucy and Laurendeau, 1995]. However, for a hand-held range sensor the viewpoint is continually changing and does not provide a reliable estimate of measurement uncertainty. Therefore, measurement uncertainty can not be reliably estimated for each measurement point with handheld sensor data. An estimate of the rms measurement error for the sensor is obtained by capturing a sphere of known radius. This estimate can be used to evaluate the likelihood of overlapping surface measurements corresponding to the same surface.

The overlap tests are applied to determine if the intersecting volumetric envelopes for meshes, $M_{0}, \ldots, M_{N}$, correspond to measurements of the same surface region. The field functions, $f_{i}(\vec{x}) a n d f_{j}(\vec{x})$, for measurements corresponding to the same surface region are combined according to a weighted average [Curless and Levoy, 1996 Hilton et al., 1996] to obtain a single implicit surface representation, $f_{i}(\vec{x})=0$. Having constructed a fused implicit surface an explicit mesh is obtained using a standard implicit surface polygonization algorithm such as Marching Cubes [Bloomenthal, 1994]

To achieve computationally efficient performance of the geometric fusion algorithm a discrete volumetric grid is used [Curless and Levoy, 1996, Hilton et al., 1998]. This enables reconstruction of a highresolution model of complex objects consisting of hundreds of thousands of polygons in a few minutes. Figure 2(b) shows the raw 3D stripe data captured using a hand-held 3D sensor. Figure 2(c) illustrates a 


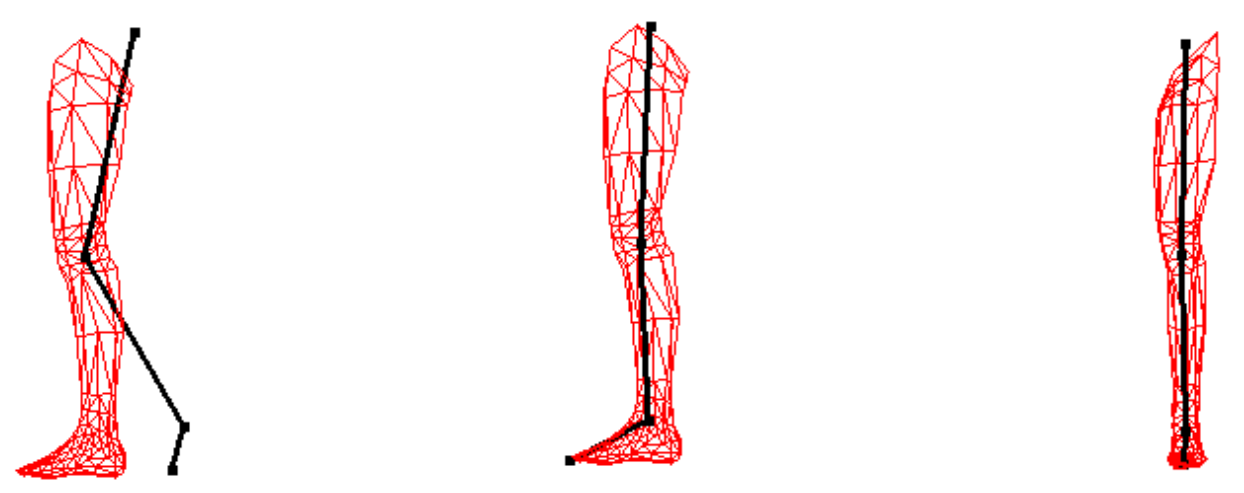

Figure 5: Skeleton fitting

set of overlapping constrained triangular patches drawn from hand-held 3D sensor data. A rendering of the high-resolution model obtained is shown in Figure 2(d).

\section{Layered Model Reconstruction}

Typically captured models are composed of millions of polygons and the articulation structure of the object is unknown. To animate captured models directly is expensive and it is difficult to achieve a satisfactory result. There are many different tasks such as motion control and realistic deformation of the model involved in designing animation. To enable realistic animation, a layered model is built in our approach. In this section we introduce a semi-automatic method for rapidly constructing an animated layered model from captured data. Initially, a skeleton is manually placed inside the model. Automatic mapping is then performed to attach points on the low-resolution control model to the skeleton and high-resolution data to the low-resolution control model. The different animation tasks involved are decomposed into sub-tasks lying in different layers. This process achieves reconstruction of a layered animation model in a few minutes.

\subsection{Skeleton fitting}

Using skeleton models in motion control design is a common practice for character animation. The skeleton is composed of a hierarchy of joints with linear segments attaching parent and child joints. It reflects the structure of an articulated figure and enables real-time manipulation of the articulation structure to define believable animations.

To fit the skeleton to the captured model requires joint positions to be identified. In our system a 
manual process is used to position the skeleton inside both the captured data and the low-resolution control model. An algorithm that allows the user to identify the joint positions on the screen using the mouse is developed. This is achieved by manipulating the skeleton joint positions in two orthogonal views following the hierarchical order of the skeleton structure, as illustrated in Figure 5. This allows the skeleton joint locations to be moved so that the joints are in approximately the correct positions and the segment links between joints run along the principal axes of the data. This manual skeleton positing is used as the basis for automatic mapping to form a layered animation model.

\subsection{Low-resolution model to skeleton mapping}

After fitting a predefined skeleton to the mesh model, we need an approach that can attach 3D points on the control model to the skeleton. To produce a realistic animation and enable efficient construction, we require that this 3D point-to-line mapping is automatic, complete (no point are left unattached) and continuous (the neighbourhood of a point on the mesh is mapped onto the neighbourhood of the point's corresponding point on the skeleton). The mapping is based on a single pose of the object. To enable animation of the control model from the skeleton animation, the mapping of a point to a skeleton segment is parameterised in terms of the joint angles for both ends of the segment. In our previous work [Sun et al., 1999] we used a mapping which which could not cope with twist motion of the segment. In this paper we introduce a new point-to-line mapping which produces full deformation (twist, shear,stretch and compression) of the segment geometry based on the joint angles.

The low-resolution model mapping and animation is based on the definition of joint plane which is defined from the adjacent joint positions. For the $i^{t h}$ skeleton joint with position $\vec{o}_{i}$ we define a joint plane with normal $\vec{n}_{i}$ such that for any point $\vec{x}$ in the plane:

$$
\vec{n}_{i} \bullet\left(\vec{x}-\vec{o}_{i}\right)=0
$$

The joint plane is defined by a local coordinate system based on the postions of adjacent parent and child joints, $\vec{o}_{i-1}$ and $\vec{o}_{i+1}$, so that it bisects the joint. Figure 6 illustrates the joint planes for a section of the skeleton. The local coordinate system $\left[\vec{x}_{i}, \vec{y}_{i}, \vec{z}_{i}\right]$ where the y-axis is aligned with the plane normal, $\vec{y}_{i}=\vec{n}_{i}$ is given by:

$$
\begin{aligned}
\vec{x}_{i} & =\frac{\vec{v}_{i, i+1} \times \vec{v}_{i, i-1}}{\left\|v e c v_{i, i+1} \times \vec{v}_{i, i-1}\right\|} \\
\vec{y}_{i} & =\vec{z}_{i} \times \vec{x}_{i} \\
\vec{z}_{i} & =\frac{\vec{x}_{i} \times \vec{v}_{i, i+1}+\vec{v}_{i, i-1} \times \vec{x}_{i}}{\left\|\vec{x}_{i} \times \vec{v}_{i, i+1}+\vec{v}_{i, i-1} \times \vec{x}_{i}\right\|}
\end{aligned}
$$




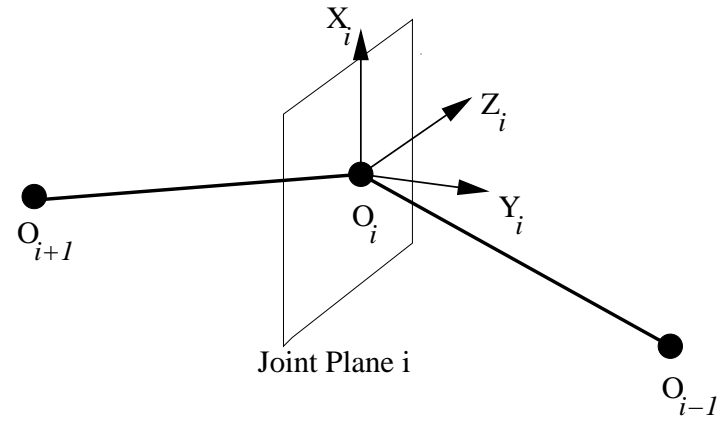

Figure 6: Joint planes and local coordinates

where $v_{i, j}=\left(\vec{o}_{j}-\vec{o}_{i}\right)$ is the vector between the $i^{t h}$ and $j^{\text {th }}$ joints and $\times$ represents the vector cross-product. In equation 4 the z-axis bisects the angle between the joints in the plane defined by the joint positions $\left(\vec{o}_{i}, \vec{o}_{i+1}, \vec{o}_{i-1}\right)$. If the joint is an end-effector having no child-joint the $\mathrm{x}$-axis is equal to the parent joint axis are defined as: $\left[\vec{x}_{i}=\vec{x}_{i-1}, \vec{y}_{i}=\vec{v}_{i, i-1}, \vec{z}_{i}=\vec{x}_{i} \times \vec{y}_{i}\right]$.

The problem of calculating which segment a vertex $\vec{p}_{j}$ shall be mapped to is converted to the problem of determining the two joint planes that the vertex lies in between [Sun et al., 1999]. After finding which segment a vertex shall be attached, we want to register it in local coordinate system in its attached segment and the segment's two joint planes. The point can then me animated according to the movement of the joint plane from skeletal animation resulting in a corresponding deformation of the control mesh. Let $i$ and $i+1$ be the indices of joint planes for a segment, as illustrated in Figure 7. Consider a line $S$ which intersects point $\vec{p}_{j}$ and is parallel to segment vector $\vec{v}_{i, i+1}$. The points $\vec{p}_{j} i$ and $\vec{p}_{j i+1}$ are the intersections of line $S$ with the joint planes at $i$ and $i+1$. Then normalised vectors $\vec{v}_{j} i=\left(\operatorname{vecp}_{j i}-v_{e c o_{i}}\right) /\left|\operatorname{vecp}_{j i}-v_{e c o_{i}}\right|$ and $\vec{v}_{j i+1}=\left(\operatorname{vecp}_{j i+1}-v_{e c o_{i+1}}\right) / \mid$ vecp $_{j i+1}-v_{e c o_{i+1}} \mid$ can be expressed in terms of the corresponding joint plane coordinate system as:

$$
\begin{aligned}
\vec{v}_{i} & =\cos \theta_{i} \vec{z}_{i}+\sin \theta_{i} \vec{x}_{i} \\
\vec{v}_{i+1} & =\cos \theta_{i+1} \vec{z}_{i+1}+\sin \theta_{i+1} \vec{x}_{i+1}
\end{aligned}
$$

where $\theta_{i}$ is the angle between $\vec{v}_{i}$ and $\vec{z}_{i}$. The mapping for vertex $\vec{p}_{j}$ to segment $(i, i+1)$ is given by:

$$
\vec{p}_{j}=\alpha \vec{o}_{i}+(1-\alpha) \vec{o}_{i+1}+d\left(\alpha \vec{n}_{i}+(1-\alpha) \vec{n}_{i+1}\right)
$$

alpha $=\mid\left(p_{j}-p_{j i}\right)\|/\|\left(p_{j i+1}-p j\right) \|$. This mapping represents a point $p_{j}$ attached to segment $(i, i+1)$ 


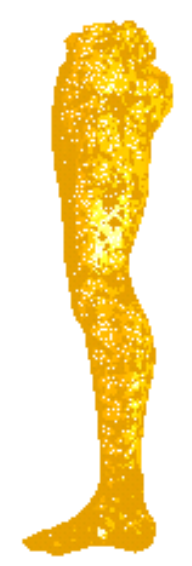

(a)

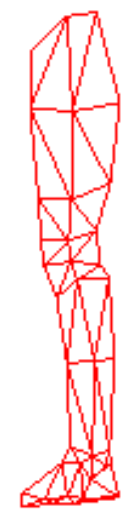

(b)

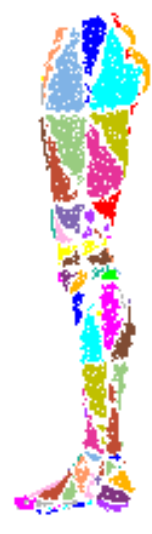

(c)

Figure 9: Mapping high-resolution leg model to low-resolution leg model

and $\vec{p}_{3}=\left(\vec{v}_{t}+d \vec{n}_{t}\right)$. The plane equation can be written as:

$$
\vec{n} \bullet \vec{x}=\vec{n} \bullet \overrightarrow{p_{1}}
$$

where $\vec{n}=\vec{v}_{12} \times \vec{v}_{13}$ and $\vec{v}_{i j}=\vec{p}_{j}-\vec{p}_{i}$. This formulation is used to solve for $d$. Figure 8 illustrates the plane through the point $\vec{x}$ defined by equation 9 . The plane is defined by three points $\left(\vec{p}_{1}, \vec{p}_{2}, \vec{p}_{3}\right)$ which are displacements by a distance $d$ along the vertex normals. It should be noted that each value of $d$ defines a unique plane. The union of these planes is a continuous volume which encloses all points in 3-space. Having obtained a solution for the variable $d$, the coefficients $\alpha$ and $\beta$ can be evaluated from equation 8 .

Figure 9 illustrates the mapping of the high-resolution captured data model (Figure 9(a)) to a lowresolution control model (Figure 9(b)). The results of the mapping process are shown in Figure 9(c). Each triangle on the high-resolution model is coloured according to the low-resolution triangle to which it is mapped. Triangles with vertices mapping to more than one low-resolution triangle are coloured black for visualisation purpose. It should be noted that all mesh vertices are mapped correctly including those belonging to triangles which cross low-resolution triangle borders. This result clearly illustrates that pointto-surface mapping partitions the 3D space to map 3D vertices on the high-resolution mesh to the lowresolution control mesh. This mapping can be performed for arbitrary geometry of the high-resolution surface to obtain a local parameterisation of measurement data.

The mapping does not require that the low-resolution model is a good approximation to the highresolution data as the normal-volumes fill the entire 3D space around the low-resolution model. Figure 10 illustrates the continuous mapping of a detailed head shape to a simple cube composed of twelve trian- 


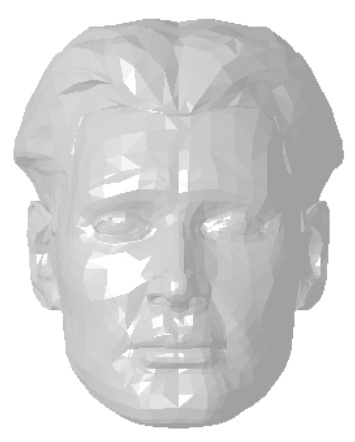

(a) High-resolution Model

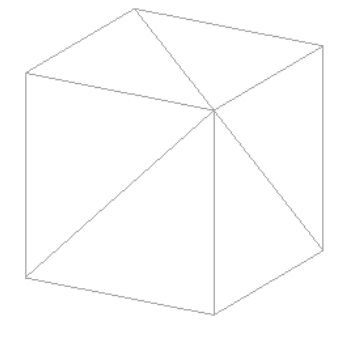

(b) Low-Resolution Control Model

(c) Normal-Volume Mapping

Figure 10: Mapping high-resolution head to a simple low-resolution cube model

gles. To achieve realistic animation of the high-resolution model based on deformation of the low-resolution model it is necessary to structure the low-resolution model to give a smooth change in shape during animation.

\section{Layered Model Animation}

The mappings of the low-resolution model triangles to the skeleton and high-resolution captured model to the low-resolution model enable us to animate the low-resolution and high-resolution models. This section will discuss how these models are animated.

\subsection{Seamless deformation of control mesh}

In section 4.2, we have introduced the approach used for mapping points to lines. Given a generic model, after fitting a skeleton to it, the mapping algorithm attaches the mesh of the generic model to the skeleton automatically. This mapping is performed only for a single pose of the generic model. How does the model deform when the skeleton moves? The objective here is to achieve a seamless and realistic deformation of the generic model during animation. Geometric mesh deformation has previously been used for seamless animation [Babski and Thalmann, 1999b, Kalra et al., 1998]. In this section we show how the point-to-line mapping and parameterisation presented in section 4.2 can be used to animate an arbitrary geometry and topology low-resolution model.

For each pose of the skeleton during the movement, we first calculate the orientations of joint planes as discussed in section 4.2. Then for each vertex in the generic model we use the mapping results and joint 

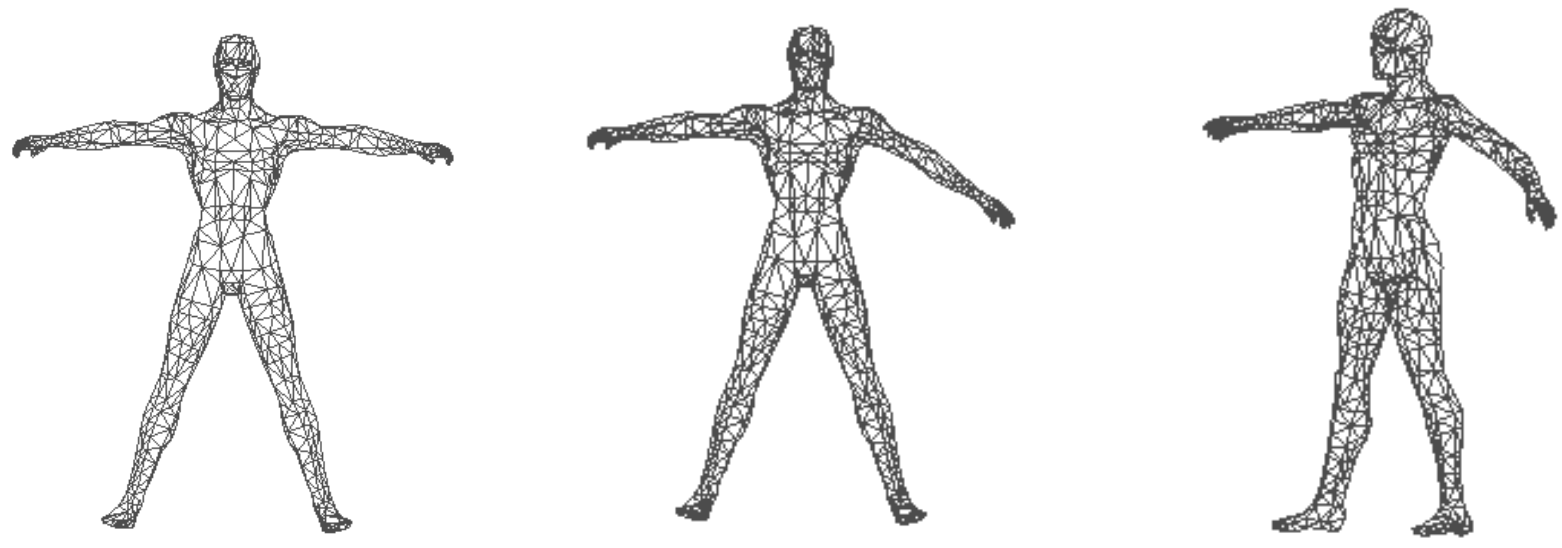

Figure 12: Animation of whole body: low-resolution control model
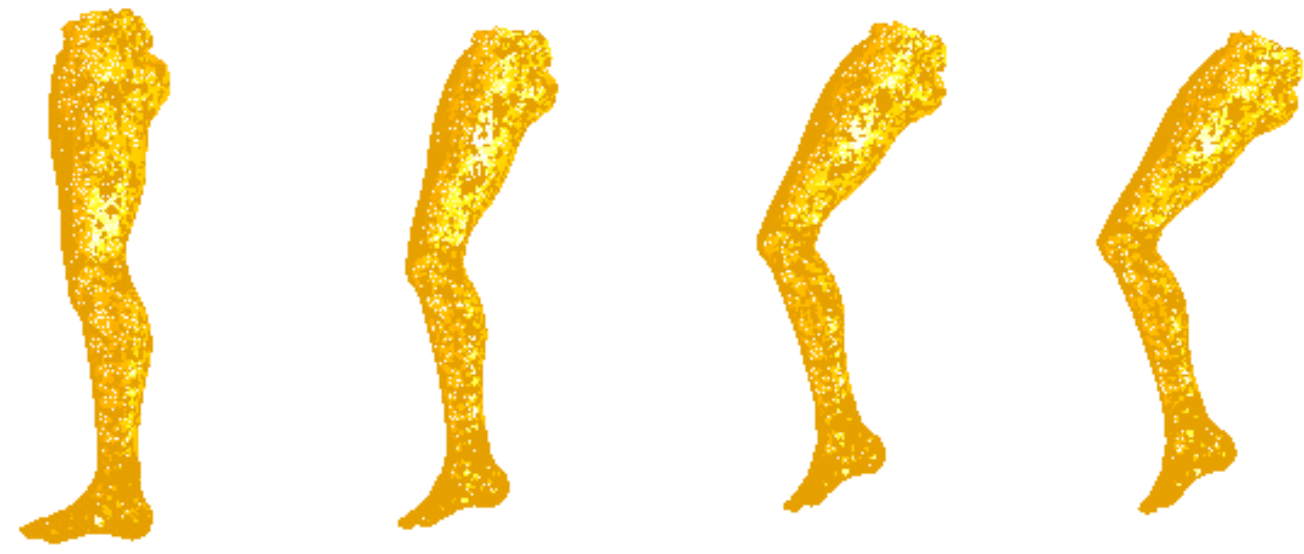

Figure 13: Layered animation of high-resolution captured data model for leg 

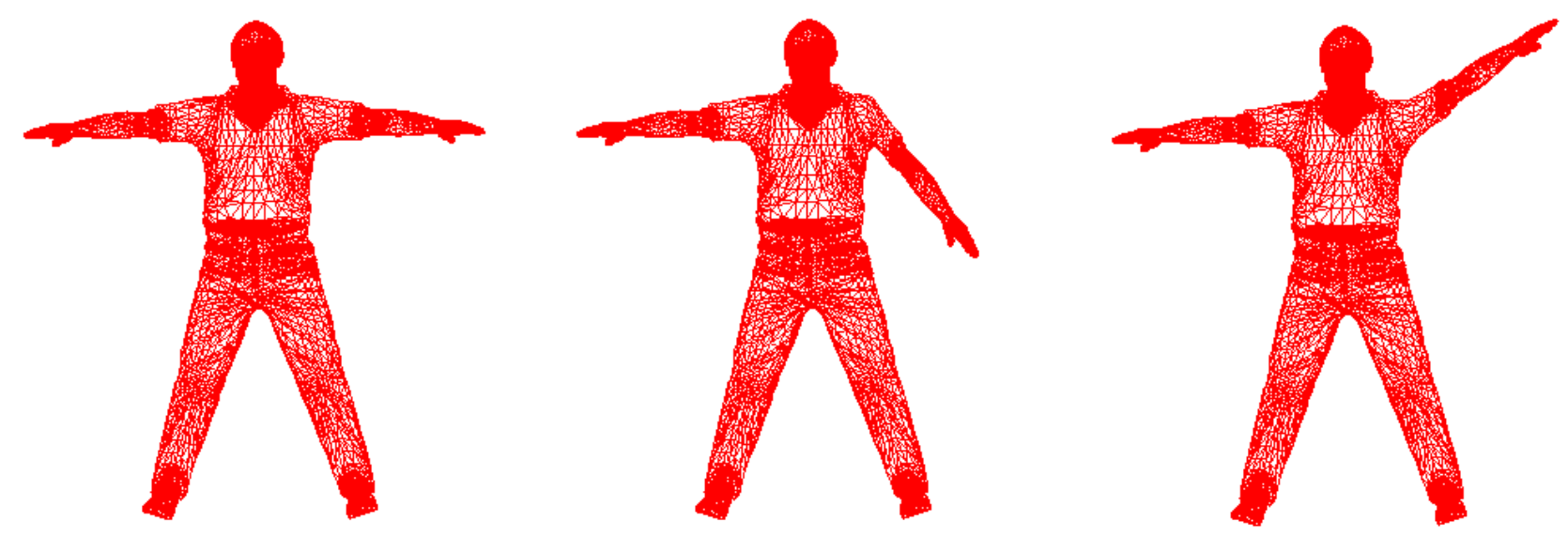

Figure 14: Whole body's arm animation of high-resolution model

Given a high-resolution model $M=\{P, T\}$ we pre-compute for each mesh vertex $\vec{x}$ the nearest mapping inside the normal-volume of a low-resolution triangle $t_{j}$ with mapping coefficients $(\alpha, \beta, d)$ as discussed in section 4.3. This has been shown in Figure 8(b). If the low-resolution model geometry is modified such that vertices of triangle $t_{j}$ are changed to new positions $\left(\vec{v}_{\text {newr }}, \vec{v}_{n e w s}, \vec{v}_{n e w t}\right)$ and vertex normals are modified to $\left(\vec{n}_{\text {newr }}, \vec{n}_{\text {news }}, \vec{n}_{\text {newt }}\right)$. Using equation 7 and equation 8 we can evaluate the new position of vertex $\vec{x}, \vec{x}_{n e w}$, on the high-resolution model as:

$$
\begin{aligned}
\vec{p}_{\text {newj }} & =\alpha \vec{v}_{\text {newr }}+\beta \vec{v}_{n e w s}+(1-\alpha-\beta) \vec{v}_{\text {newt }} \\
\vec{n}_{\text {new }} & =\alpha \vec{n}_{n e w r}+\beta \vec{n}_{n e w s}+(1-\alpha-\beta) \vec{n}_{n e w t} \\
\vec{x}_{n e w} & =\vec{p}_{n e w j}+d \vec{n}_{n e w j}
\end{aligned}
$$

As the new vertex position $\vec{x}_{n e w}$ is only dependent on the triangle vertex positions and normals of the underlying low-resolution model, the high resolution model will deform efficiently and seamlessly as the low-resolution structure is manipulated. Figure 13, Figure 14 and Figure 15 show some results of our layered animation of high-resolution captured data models. The leg model (Figure 13) has 5102 vertices and 10200 triangles and the generic model used only has 210 vertices and 416 triangles (Figure 11(a)). Figure 14 consists of 13202 vertices and 26243 triangles and the control model has 1010 vertices and 2014 triangles. The dinosaur in Figure 15 has 17334 vertices and 36276 triangles (texture mapped model from [Fitzgibbon et al., 1998]). The results demonstrate that using our layered animation model, efficient and realistic animation of captured high-resolution data can be achieved. 
quality of the low-resolution model animation. Greater realism could be achieved using alternative methods such as FFD or physics-based techniques to animate the low-resolution model. The layered animation framework provides the flexibility to use other animation methods but this has not yet been tested.

\section{Conclusions}

In this paper we have proposed a technique for building layer animation models of real objects from 3D surface measurement data of real objects to achieve realistic animation whilst maintaining detail. A handheld range sensor system is used to capture objects. The captured models have highly detailed and realistic surfaces but are composed of millions of polygons and their articulation structures are unknown. A layered animation system is built to animate captured models realistically and efficiently. Initially a skeleton model is placed inside the captured data. Automatic mapping procedures are introduced to construct a mapping of the high-resolution model to a low-resolution model and low-resolution model to a skeleton. This procedure is quick and efficient resulting in a layered animation model which allows real-time manipulation and highresolution rendering.

The principal contributions of this paper are:

- Geometric fusion of measurements from a hand-held range sensor into a single mesh surface using a new volumetric integration algorithm based on the normal-volume.

- Introduction of a new point-to-surface mapping algorithm based on the normal-volume. This approach enables the correspondence between the high-resolution captured model and a low-resolution control model to be automatically established.

- Presentation of a layered animation framework which achieves seamless deformable animation of high-resolution captured models based on skeleton animation and deformation of a low-resolution control model.

The animation of high-resolution captured data based on a low-resolution generic model of the object opens up the possibility of being able to rapidly capture and animate new objects based on existing libraries of generic models. 


\section{References}

[Babski and Thalmann, 1999a] Babski, C. and Thalmann, D. (1999a). A seamless shape for hanim compliant bodies. In Proc. IEEE VRML Conference, pages $21-28$.

[Babski and Thalmann, 1999b] Babski, C. and Thalmann, D. (1999b). A seamless shape for hanim compliant bodies. In Proc. VRML'99, pages 21-28.

[Bittar et al., 1995] Bittar, E., Tsingos, N., and Gascuel, M.-P. (1995). Automatic reconstruction of unstructured 3d data: Combining a medial axis and implicit surface. Computer Graphics Forum, 14(3):458468.

[Bloomenthal, 1994] Bloomenthal, J. (1994). An implicit surface polygonizer. Graphics Gems ed. Heckbert,P.S., 4:324-350.

[Bloomenthal and Wyvill, 1990] Bloomenthal, J. and Wyvill, B. (1990). Interactive techniques for implicit modelling. ACM Computer Graphics, 24(2):109-116.

[Chadwick and Parent, 1989] Chadwick, J. Haumann, D. R. and Parent, R. E. (1989). Layered construction for deformable animated characters. ACM Computer Graphics, 23(3):234-243.

[Chang and Rockwood, 1994] Chang, Y. and Rockwood, A. (1994). A generalized de casteljau approach to 3d free-form deformation. ACM Computer Graphics, 24(4):257-260.

[Cohen et al., 1996] Cohen, J., Varshney, A., Manocha, D., Turk, G., Weber, H., Agarwal, P., Brooks, F., and Wright, W. (1996). Simplification Envelopes. In ACM Computer Graphics Proc., SIGGRAPH, NewOrleans, USA, pages 119-128.

[Coquillart and Jancene, 1991] Coquillart, S. and Jancene, P. (1991). Animated free-form deformations: An interactive animation techniques. In ACM SIGGRAPH, pages 23-26.

[Curless and Levoy, 1996] Curless, B. and Levoy, M. (1996). A Volumetric Method for Building Complex Models from Range Images. In ACM Computer Graphics Proceedings, SIGGRAPH, NewOrleans, USA, pages $303-312$.

[Datalabs, 1996] Datalabs, V. (1996). Viewpoint Catalog. (http://www.viewpoint.com). 
[Eck et al., 1995] Eck, M., DeRose, T., Duchamp, T., Hoppe, H., Lounsbery, M., and Stuetzle, W. (1995). Multiresolution analysis of arbitary meshes. In SIGGRAPH, pages 173-182.

[Eck and Hoppe, 1996] Eck, M. and Hoppe, H. (1996). Automatic reconstruction of b-spline surfaces of arbitrary topological type. In Proc. ACM SIGGRAPH, pages 325-334.

[Edelsbrunner and Mucke, 1994] Edelsbrunner, H. and Mucke, E. (1994). Weighted alpha shapes. ACM Transactions on graphics, 13(1):43-72.

[Fitzgibbon et al., 1998] Fitzgibbon, A. W., Cross, G., and Zisserman, A. (1998). Automatic 3D model construction for turn-table sequences. In Koch, R. and Van Gool, L., editors, 3D Structure from Multiple Images of Large-Scale Environments, LNCS 1506, pages 155-170. Springer-Verlag.

[Forsey, 1991] Forsey, D. R. (1991). A surface model for skeleton-based character animation. In Proc.Second Eurographics Workshop on Animation and Simulation, pages 55-74.

[Hilton et al., 1996] Hilton, A., Stoddart, A., Illingworth, J., and Windeatt, T. (1996). Reliable surface reconstruction from multiple range images. In 4th European Conference on Computer Vision, pages 117-126. Springer.

[Hilton et al., 1998] Hilton, A., Stoddart, A., Illingworth, J., and Windeatt, T. (March 1998). Implicit surface based geometric fusion. International Journal of Computer Vision and Image Understanding, Special Issue on CAD Based Vision, 69(3):273-291.

[Hoppe, 1996] Hoppe, H. (1996). Progressive meshes. In Proc. ACM SIGGRAPH, pages 99—108.

[Hoppe et al., 1994] Hoppe, H., DeRose, T., Duchamp, T., Halstead, M., Hin, H. McDonald, J., Schweitzer, J., and Stuetzle, W. (1994). Piecewise smooth surface reconstruction. In Computer Graphics Proceedings, SIGGRAPH, pages 295-302.

[Hoppe et al., 1992] Hoppe, H., DeRose, T., Duchamp, T., McDonald, J., and Stuetzle, W. (1992). Surface reconstruction from unorganised points. Computer Graphics, 26(2):71—77.

[Hsu et al., 1992] Hsu, W., Hughes, J., and Kaufman, H. (1992). Direct manipulation of free-form deformations. ACM Computer Graphics, 26(2):177-184. 
[Kalra et al., 1998] Kalra, N., Magnenat-Thalmann, N., Moccozet, L., Sannier, G., Aubel, A., and Thalmann, D. (1998). Real-time animation of virtual humans. IEEE Computer Graphics and Applications, $18(5): 42-56$.

[Kalra and Magnenat Thalmann, 1998] Kalra, P. and Magnenat Thalmann, N. (1998). Real-time animation of realistic virtual human. IEEE Computer Graphics and Applications, 18(5):42—56.

[Kalvin and Taylor, 1996] Kalvin, A. and Taylor, R. (1996). Superfaces: polygonal mesh simplification with bounded error. IEEE Computer Graphics and Applications, 16(3):64—77.

[Krishnamurthy and Levoy, 1996] Krishnamurthy, V. and Levoy, M. (1996). Fitting smooth surfaces to dense polygon meshes. In In ACM Computer Graphics Proc. SIGGRAPH, NewOrleans, USA.

[Lamousin and Waggenspack, 1994] Lamousin, H. J. and Waggenspack, W. N. (1994). Nurbs-based freeform deformations. IEEE Ccmputer Graphics and Applications, 14(16):59—65.

[Lander, 1998] Lander, J. (1998). Skin them bones: Game programming for the web generation. Game Developer Magazine, May.

[Lazarus and Coquillart, 1994] Lazarus, F. and Coquillart, S. adn Jancene, P. (1994). Axial deformations: An intuitive deformation technique. Computer-Aided Design, 26(8):607—613.

[MacCracken and Joy, 1996] MacCracken, R. and Joy, K. (1996). Free-form deformations with lattices of arbitrary topology. In ACM SIGGRAPH, pages 181-188.

[Maestri, 1996] Maestri, G. (1996). Digital Character Animation. New Riders Publishing, Indiana, USA.

[Mencl, 1995] Mencl, R. (1995). A graph-based approach to surface reconstruction. In EUROGRAPHICS 14(3), pages 446-456.

[Mencl and Muller, 1998] Mencl, R. and Muller, H. (1998). Graph-based surface reconstruction using structures in scattered point sets. In Computer Graphics International, pages 298-311.

[Mocozzet and Mangnenat Thalmann, 1997] Mocozzet, L. and Mangnenat Thalmann, N. (1997). Dirichlet free-form deformations and their applications to hand simulation. In IEEE Int.Conf. on Computer Animation. 
[Rutishauser et al., 1994] Rutishauser, M., Stricker, M., and Trobina, M. (1994). Merging range images of arbitrarily shaped objects. In Proceedings of IEEE Conference on Computer Vision and Pattern Recognition, pages 573-580.

[Schroeder et al., 1992] Schroeder, W., Zarge, J., and Lorensen, W. (1992). Decimation of triangular meshes. In SIGGRAPH 26(2), pages 65-70.

[Shen and Thalmann, 1995] Shen, J. and Thalmann, D. (1995). Interactive shape design using metaballs and splines. In Proc. IEEE Implicit Surfaces Conference, pages 187-196.

[Singh and Fiume, 1998] Singh, K. and Fiume, E. (1998). Wires: A geometric deformation technique. In ACM SIGGRAPH, pages 405-414.

[Soucy and Laurendeau, 1995] Soucy, M. and Laurendeau, D. (1995). A general surface approach to the integration of a set of range views. IEEE Trans. Pattern Analysis and Machine Intelligence, 14(4):344358.

[Soucy and Laurendeau, 1996] Soucy, M. and Laurendeau, D. (1996). Multiresolution surface modeling based on hierarchical triangulation. Computer Vision and Image Understanding, 63(1):1—14.

[Sun et al., 1999] Sun, W., Hilton, A., Smith, R., and Illingworth, J. (September 1999). Building layered animation models from captured data. In Eurographics Workshop on Computer Animation, pages 145154.

[Taubin and Ronfard, 1996] Taubin, G. and Ronfard, R. (1996). Implicit simplical models for adaptive curve reconstruction. IEEE Trans. Pattern Analysis and Machine Intelligence, 18(3):321-325.

[Thalmann et al., 1996] Thalmann, D., Shen, J., and Chauvineau, E. (1996). Fast realistic human body deformations for animation and vr applications. In Proc. Computer Graphics International Conference, pages $166-174$.

[Turk and Levoy, 1994] Turk, G. and Levoy, M. (1994). Zippered Polygon Meshes from Range Images. In ACM Computer Graphics Proceedings, SIGGRAPH, Orlando, Florida, pages 311-318.

[Turner and Gobbetti, 1998] Turner, R. and Gobbetti, E. (1998). Interactive construction and animation of layered elastically deformable characters,. Computer Graphics Forum, 17(2):135—152. 